\title{
The Use of Hierarchical Sampling in the Surveillance Program for Plum pox virus Incidence in the United States
}

\author{
G. Hughes, Institute of Ecology and Resource Management, University of Edinburgh, West Mains Road, Edinburgh \\ EH9 3JG, UK; T. R. Gottwald, USDA, Agricultural Research Service, Ft. Pierce, FL 34945; and L. Levy, USDA- \\ APHIS-PPQ, Center for Plant Heath Science and Technology, Beltsville, MD 20705
}

\begin{abstract}
Hughes, G., Gottwald, T. R., and Levy, L. 2002. The use of hierarchical sampling in the surveillance program for Plum pox virus incidence in the United States. Plant Dis. 86:259-263.

Plum pox virus (PPV) was first reported in North America in late 1999. As part of the eradication effort mounted in response to this finding, a Plum Pox Virus National Surveillance Program has been implemented in the United States. The survey method adopted for this program is a form of "group testing" that utilizes relationships between disease incidence at two levels in a spatial hierarchy, known as hierarchical sampling. Leaf material is collected from groups of four trees and tested by enzyme-linked immunosorbent assay (ELISA) for PPV infection without distinguishing between the individual trees in a group. PPV incidence at the scale of the individual tree is then calculated by means of a formula. Incidence data were collected during an initial survey conducted by the Pennsylvania Department of Agriculture from nine PPV-infected orchards in the fall of 1999 and from an additional 11 PPV-infected orchards during the spring and summer of 2000. The pattern of PPV-infected trees at the within-group scale was weakly aggregated. As a result, an effective sample size of $\hat{v}=3.2$, rather than the nominal group size of four, should be used in the formula to calculate an estimate of incidence at the scale of the individual tree from observations of incidence collected at the group scale.
\end{abstract}

Additional keywords: aphid vectors, Prunus, sharka, stone fruit

The first report of Plum pox virus (PPV) in North America followed the observation of PPV-like symptoms in peach fruit culls in a packing house in Pennsylvania in September 1999 (13). All symptomatic fruit originated from a single block of peach (Prunus persica cv. Encore) in Adams County (13). In response to the discovery of PPV infection in Adams County, a Plum Pox Virus National Surveillance Program was implemented in the United States. The survey method adopted for this program was based on survey methods for assessment of Citrus tristeza virus incidence developed by Hughes and Gottwald $(6,7)$, and known as "hierarchical sampling." The U.S. Plum Pox Virus National Surveillance Program guidelines (18) were completed in March 2000 and have been implemented in several states where stone fruits are grown on a commercial scale. At the time of writing (November 2000), the only reports of PPV infection in the United States have come from orchards in Pennsylvania. In

Corresponding author: G. Hughes

E-mail: ghughes@srv0.bio.ed.ac.uk

Accepted for publication 22 October 2001.

Publication no. D-2002-0118-01R

This article is in the public domain and not copyrightable. It may be freely reprinted with customary crediting of the source. The American Phytopathological Society, 2002.
June 2000, the Canadian Food Inspection Agency (CFIA) confirmed the presence PPV infection in an orchard in Ontario. Additional PPV-positive sites have subsequently been identified in Ontario and Nova Scotia, using a survey method similar to that of the U.S. Plum Pox Virus National Surveillance Program.

Hierarchical sampling is a form of "group testing" that utilizes relationships between disease incidence at two levels in a spatial hierarchy (11). Material is collected from groups of trees and assayed without distinguishing among individual trees in the group. Testing material from groups of trees saves time and labor (20) and so makes efficient use of resources devoted to sampling. Incidence at the scale of the individual tree is calculated by means of a formula that involves incidence at the scale of the group and group size. In conventional group testing, material collected from individuals is assigned to groups at random, prior to testing in the laboratory, and the disease status of one member of a group may therefore be assumed to be independent of the disease status of other members of the same group. In such circumstances, a formula based on the zero term of the binomial distribution may be assumed to be appropriate for the calculation of incidence at the scale of the individual tree. In the hierarchical sampling scheme, an orchard block is thought of as being divided into groups of four trees, each group in a two-by-two rectangular arrangement. Beginning at the corner of the block to be sampled, one out of the first four groups is selected at random, then every fourth group of four systematically after that (see Figure 1 in Hughes and Gottwald [7]). In hierarchical sampling, the locations of sampled groups are recorded, so it is possible to return to a group found to be PPV positive either to re-test the individual trees (if a regulatory policy of only removing infected trees is in place) or to remove all the trees in the group (if that is the policy). When a group comprises a number of spatially adjacent trees, the virus status of one member of a group cannot be assumed to be independent of the virus status of other members of the same group. Because the virus status of a tree may not be independent of the virus status of its neighbors, use of a formula based on the binomial distribution may not be justified. An analysis that takes into account the pattern of infected trees at the within-group scale is required.

The objective of this article is to present the epidemiological data analysis that provides the scientific background to the adoption of the hierarchical sampling method for PPV incidence in the U.S. Plum Pox Virus National Surveillance Program.

\section{MATERIALS AND METHODS}

Laboratory assay for PPV. Laboratory protocols for PPV detection by enzymelinked immunosorbent assay (ELISA) are described by Levy (12). A prerequisite for the implementation of a group testing procedure such as hierarchical sampling is that the laboratory assay must be capable of detecting a single infected individual in a group. To test this, leaf lamina disks were removed from both healthy and infected greenhouse-grown Stanley plum (Prunus domestica); total weight was $0.3 \mathrm{~g}$ of leaf tissue. The leaf tissue was ground at a dilution of 1:10 in PPV extraction buffer. ELISA for PPV detection was conducted according to the kit manufacturers' recommendations (Agdia Inc., Elkhart, IN, and Durviz S. L., Valencia, Spain) with the following modification. A dilution series of $16,14,12,10,8,6,5,4,3,2,1$, and 0 (control) PPV-positive leaf disks out of a total of 16 disks was tested against a dilution series of the PPV-specific monoclonal antibody 5B-IVIA (component A2 of the 
ELISA kit) as follows: 1:500, 1:1,000, 1:20,000 PPV-antiserum:buffer. ELISA reactions were incubated with alkaline phosphatase substrate at room temperature. Two duplicate wells were used per sample. After $1 \mathrm{~h}$ of incubation, absorbances were measured at $405 \mathrm{~nm}$ in an ELISA reader (Molecular Devices Corporation, Sunnyvale, CA). A sample was considered PPVpositive if the absorbance at $405 \mathrm{~nm}$ of the the healthy tissue control. The experiment

Initial field survey. Following the confirmation of PPV infection in Adams County, an initial survey was conducted by the Pennsylvania Department of Agriculnumber of orchard blocks with visual symptoms of PPV infection were located before leaf fall made further sampling impossible. Plots consisting of 400 trees in a 20 by 20 rectangular pattern were established in nine of these blocks (i.e., all those of sufficient size). In these plots, the location and PPV status (determined by ELISA) of each tree were recorded in the form of a "map." Missing trees were also recorded. More orchard blocks with visual symptoms of PPV infection were located when the survey was continued in the spring and summer of 2000. Using the same protocol as outlined above, an addi$1: 2,000, \quad 1: 4,000, \quad 1: 8,000, \quad 1: 10,000$, reaction was greater than 2.5 times that of was repeated. ture in the fall of 1999. In this survey, a

tional 11 plots were then mapped. These data, from 20 plots in all, provide a basis for the analysis of a relationship between the incidence of PPV infection at two spatial scales that underlies the hierarchical sampling method.

Characterizing a relationship between incidence at two spatial scales. Calculations relating to the lower of the two spatial scales (in this case, the individual tree scale) are denoted by the subscript "low," while those relating to the higher of the two scales (the group scale) are denoted by the subscript "high." Consider a set of orchard blocks, in each of which a plot has been assessed for PPV (each plot assessed for PPV is referred to as a "batch"). The $j$ th batch $(j=1,2, \ldots, M$; in the present case, $M$ =20) comprises $N_{j}$ sampling units (a sampling unit is referred to as a "group"). In the $i$ th group $\left(i=1,2, \ldots, N_{j}\right)$ of the $j$ th batch, there are $n_{i j}$ trees, of which $X_{l o w, i j}$ are PPV-positive. The proportion of PPV-positive trees in the $i$ th group of the $j$ th batch is:

$$
\hat{p}_{l o w, i j}=X_{l o w, i j} / n_{i j}
$$

The mean proportion of PPV-positive trees in the $j$ th batch is:

$$
\hat{p}_{l o w, j}=\sum_{i} X_{l o w, i j} / \sum_{i} n_{i j}
$$

which is an estimate of $p_{\text {low }, j}$, the unobserved probability that a tree in the $j$ th

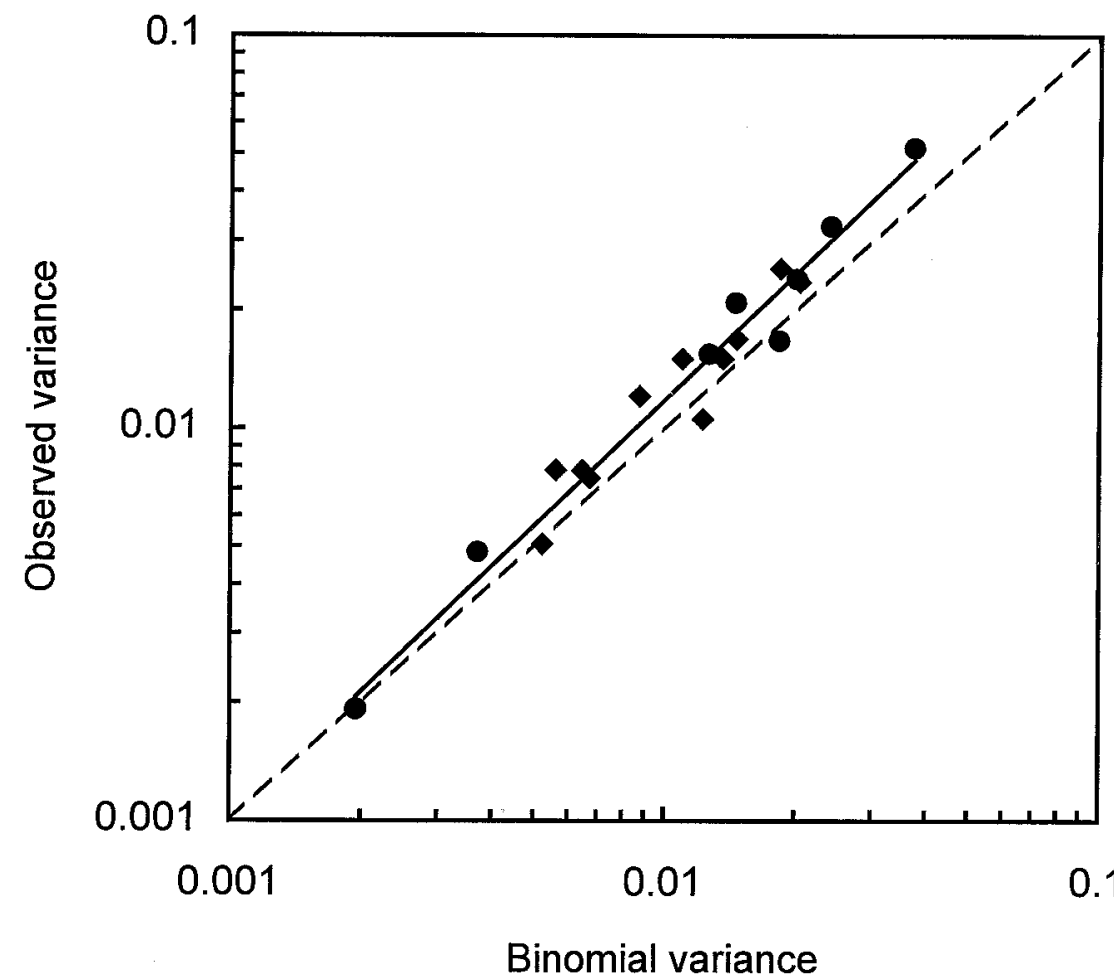

Fig. 1. Relationship between the observed and the theoretical binomial (random) variances of incidence of Plum pox virus (PPV) infection at the individual tree scale (note the use of logarithmic scales on both axes). Each data point represents a PPV assessment in a stone fruit orchard block in Adams County, PA (•, assessed in fall 1999; • assessed in spring/summer 2000). The solid line represents the relationship $\log \left(v \hat{a} r_{\text {low }}\right)=0.20+1.06 \cdot \log \left(v \hat{a} r_{\text {bin,low }}\right)$, fitted to the data by ordinary least squares regression analysis. The dashed line $(---)$ represents the binomial line (i.e., observed variance $=$ binomial variance) . batch is PPV-positive. This represents disease incidence at the scale of the individual tree. The mean group size in the $j$ th batch is:

$$
n_{j}=\sum_{i} n_{i j} / N_{j}
$$

and the mean group size over all batches is:

$$
n=\sum_{i j} n_{i j} / \sum_{j} N_{j}
$$

In the present study, the nominal group size is four trees, in a two-by-two rectangular arrangement. If there are missing trees, the calculated mean group sizes will be smaller than the nominal group size.

For the analysis of the PPV data presented here, a grid representing $N_{j}=100$ groups of four trees was superimposed on the map for each batch, and each group assessed as PPV-positive (a group containing at least one PPV-positive tree) or PPVnegative (a group containing no PPV-positive trees). In the $j$ th batch, $X_{h i g h, i j}=1$ if the $i$ th group is classified as PPV-positive or $X_{\text {high,ij }}=0$ if it is classified PPV-negative. Thus, in the $j$ th batch there are:

$$
X_{h i g h, j}=\sum_{i} X_{h i g h, i j}
$$

PPV-positive groups. The proportion of PPV-positive groups in the $j$ th batch is:

$$
\hat{p}_{h i g h, j}=X_{h i g h, j} / N_{j}
$$

which is an estimate of $p_{h i g h, j}$, the unobserved probability that a group in the $j$ th batch contains at least one diseased tree. This represents disease incidence at the group scale.

The $X_{l o w, i j}, n_{i j}$ data were used to calculate the (approximate) observed variances of PPV incidence:

$$
v \hat{a} r_{\text {low }, j} \approx \frac{\sum_{j} n_{i j}^{2}\left(\hat{p}_{\text {low }, i j}-\hat{p}_{\text {low }, j}\right)^{2}}{n_{j}^{2}\left(N_{j}-1\right)}
$$

(3) and the corresponding binomial (random) variances:

$$
v \hat{a} r_{b i n, l o w, j}=\frac{\hat{p}_{l o w, j}\left(1-\hat{p}_{l o w, j}\right)}{n_{j}}
$$

A graphical plot of observed variance against binomial variance, with logarithmic scales on both axes, was calculated (8), and the slope and intercept of the relationship between $\log \left(v \hat{a} r_{\text {low }}\right)$ and $\log \left(v \hat{a} r_{\text {bin,low }}\right)$ were estimated by ordinary least-squares regression. The relationship:

$$
\log \left(v \hat{a} r_{\text {low }}\right)=\log (\hat{A})+\hat{b} \cdot \log \left(v \hat{a} r_{\text {bin,low }}\right)
$$

and its equivalent on untransformed axes:

$$
v \hat{a} r_{l o w}=\hat{A} \cdot\left(v \hat{a} r_{b i n, l o w}\right)^{\hat{b}}
$$

amount to a description of the pattern of PPV-positive trees at the within-group scale, in terms of either the binomial or the beta-binomial distribution, depending on 
the values of the estimates $\hat{A}$ and $\hat{b}$ $(9,14)$. The values $\hat{A}=1, \hat{b}=1$ correspond to a random pattern described by the binomial distribution. If the position of the fitted regression line is above the theoretical "binomial line" (i.e., observed variance $=$ binomial variance), this is indicative of aggregation at the within-group scale that can be described by the beta-binomial distribution $(9,14)$.

The same $X_{l o w, i j}, n_{i j}$ data were analyzed using the methodology described by Hughes and Madden (10). "Batch" (a plot assessed for PPV within an orchard block) was declared a factor with 20 levels. The $X_{l o w, i j}, n_{i j}$ data were fitted to a linear logistic model with a separate location parameter for each batch, then to a beta-binomial model with a separate location parameter for each batch and a single aggregation parameter (using EGRET for Windows version 2.0, Cytel Software Corporation, Cambridge, MA). Fitting a linear logistic model in this way amounts to fitting binomial distributions to the frequency distributions of PPV-positive trees per group, allowing a different mean incidence for each batch (10). Fitting a beta-binomial model amounts to fitting beta-binomial distributions to the frequency distributions of PPVpositive trees per group, allowing a different mean incidence for each batch and an estimate of the aggregation parameter $(\hat{\theta})$ common to all batches (10). Similar analyses were also carried out separately for the subset of the data collected during the fall of 1999 (in which batch is a factor with nine levels) and for the subset collected during the following spring and summer (in which batch is a factor with 11 levels).

A graphical plot of PPV incidence at the group scale against PPV incidence at the tree scale was calculated. For a random pattern of PPV-positive trees at the withingroup scale, a formula based on the zero term of the binomial distribution may be used to describe the data plotted in this way (15). In this case, the probability that a group contains at least one PPV-positive tree $\left(p_{\text {high }}\right)$ is related to the probability that an individual tree is positive $\left(p_{\text {low }}\right)$ by: $p_{\text {high }}=1-\left(1-p_{\text {low }}\right)^{n}$

For data that are aggregated at the withingroup scale, the points $\left(\hat{p}_{\text {low }, j}, \hat{p}_{\text {high, }}\right)$ fall mostly below the curve represented by equation 1 . From a sampling perspective, the implication of this is that if the straightforward rearrangement of equation 1 :

$$
p_{\text {low }}=1-\left(1-p_{\text {high }}\right)^{\frac{1}{n}}
$$

were used as a basis for estimating disease incidence at the scale of the individual tree from observed data for incidence at the group scale:

$$
\tilde{p}_{\text {low }}=1-\left(1-\hat{p}_{\text {high }}\right)^{\frac{1}{n}}
$$

(a tilde being used to refer to an estimate that has been made from observations at a spatial scale other than the one denoted by its subscript), it would tend to produce underestimates.

Effective sample size. Aggregation at the within-group scale is the tendency for trees in the same group to have the same PPV status (either positive or negative). Because of this, less information about PPV incidence is obtained from withingroup replicates than would be the case if the PPV status of a tree could be regarded as independent of the PPV status of others in the same group. Essentially, aggregation means that less than four trees-worth of information is obtained from a group of four trees. In such cases, an "effective sample size" (denoted $v$, lowercase Greek "nu") can be substituted for $n$ (15). This provides a basis for a simple equation in the format of equation 1 :

$$
p_{\text {high }}=1-\left(1-p_{\text {low }}\right)^{v}
$$

that, by straightforward rearrangement, gives:

$$
p_{\text {low }}=1-\left(1-p_{\text {high }}\right)^{\frac{1}{v}}
$$

Mean incidence at the scale of the individual tree may then be estimated from observations made at the group scale from:

$$
\tilde{p}_{\text {low }}=1-\left(1-\hat{p}_{\text {high }}\right) \frac{1}{\hat{v}}
$$

The estimate of effective sample size can be calculated from equation 10 in Madden and Hughes (15) using the common betabinomial aggregation parameter estimate $(\hat{\theta})$, obtained as described above.

\section{RESULTS}

The ability of ELISA to detect various mixtures of PPV-positive and PPV-negative tissue is shown in Table 1. At the standard antiserum dilution of 1:1,000, the results show that the laboratory assay used for PPV can reliably detect the virus if there are two or more PPV-positive leaves among 16 leaves tested together (Table 1). Equivalent results (data not shown) were obtained from the repeat of the experiment, leading to the same conclusion.

Mean group size calculated for each batch was in the range $2.3 \leq n_{j} \leq 4.0$, and calculated mean group size over all batches was $n=3.7$. The graphical plot of observed variance against binomial variance, with logarithmic scales on both axes, was linear (Fig. 1) and had a slope of 1.06 ( $\mathrm{SE}=$ $0.04)$ and an intercept of $0.20(\mathrm{SE}=0.09)$. The position of the fitted regression line, slightly above the theoretical binomial line (i.e., observed variance $=$ binomial variance) (Fig. 1), is indicative of weak aggregation of PPV incidence at the withingroup scale.

Fitting a beta-binomial model with a common aggregation parameter to all the $X_{\text {low }, i j}, n_{i j}$ data, after first fitting the linear logistic model with a separate location parameter for each batch, resulted in a reduction in the residual deviance. The likelihood ratio statistic was 39.9 (1 df) $(P$ $<0.001)$. The maximum likelihood estimate of the beta-binomial aggregation parameter provided by this procedure was $\hat{\theta}=0.09$ (SE $=0.02)$, also indicative of weak aggregation of PPV incidence at the

\begin{tabular}{|c|c|c|c|c|c|c|c|c|c|c|c|c|}
\hline \multirow{2}{*}{$\begin{array}{l}\text { Antiserum } \\
\text { dilution }^{\mathrm{a}}\end{array}$} & \multicolumn{12}{|c|}{ Number of PPV-infected leaf disks/16 } \\
\hline & $16 / 16$ & $14 / 16$ & $12 / 16$ & $10 / 16$ & $8 / 16$ & $6 / 16$ & $5 / 16$ & $4 / 16$ & $3 / 16$ & $2 / 16$ & $1 / 16$ & $\mathbf{0} / 16^{b}$ \\
\hline $1 / 500$ & $0.927^{\mathrm{c}}$ & 0.866 & 0.823 & 0.686 & 0.624 & 0.589 & 0.518 & 0.466 & 0.431 & 0.303 & 0.173 & 0.077 \\
\hline $1 / 1,000$ & 0.656 & 0.671 & 0.557 & 0.524 & 0.482 & 0.451 & 0.438 & 0.420 & 0.377 & 0.275 & 0.160 & 0.076 \\
\hline $1 / 2,000$ & 0.454 & 0.465 & 0.408 & 0.370 & 0.360 & 0.320 & 0.340 & 0.327 & 0.303 & 0.221 & 0.144 & 0.074 \\
\hline $1 / 4,000$ & 0.272 & 0.257 & 0.234 & 0.220 & 0.218 & 0.196 & 0.217 & 0.212 & 0.195 & 0.160 & 0.116 & 0.070 \\
\hline $1 / 8,000$ & 0.152 & 0.156 & 0.138 & 0.136 & 0.136 & 0.140 & 0.138 & 0.133 & 0.133 & 0.113 & 0.090 & 0.071 \\
\hline $1 / 10,000$ & 0.132 & 0.126 & 0.134 & 0.118 & 0.117 & 0.114 & 0.120 & 0.117 & 0.112 & 0.101 & 0.086 & 0.071 \\
\hline $1 / 20,000$ & 0.104 & 0.099 & 0.098 & 0.092 & 0.091 & 0.091 & 0.099 & 0.092 & 0.092 & 0.085 & 0.079 & 0.075 \\
\hline Buffer & 0.074 & 0.073 & 0.072 & 0.068 & 0.074 & 0.072 & 0.074 & 0.078 & 0.071 & 0.073 & 0.072 & 0.073 \\
\hline
\end{tabular}
within-group scale. When separate analyses were carried out for the subset of the

Table 1. Detection of Plum pox virus (PPV)-positive leaf tissue by enzyme-linked immunosorbent assay (ELISA) in mixtures of PPV-positive and PPVnegative leaf lamina disks

a Dilution of PPV universal monoclonal antibody 5B-IVIA.

b Healthy control.

c Values in the body of the table are absorbance measured at $405 \mathrm{~nm}$ after $1 \mathrm{~h}$ of incubation with substrate. Two duplicate wells were used per sample. Values $>2.5 \times$ that of the healthy control are indicated in italic type. Results shown are from the first experiment. The repeat experiment gave equivalent results (data not shown). 
data collected during the fall of 1999 and for the subset collected during the following spring and summer, the maximum likelihood estimates of the beta-binomial aggregation parameter were $\hat{\theta}=0.12(\mathrm{SE}=$ 0.04 ) and $\hat{\theta}=0.08$ (SE $=0.03$ ), respectively. Since the ranges of $\hat{\theta} \pm S E$ overlap, the overall estimate of $\hat{\theta}=0.09$ was used for further analysis. PPV incidence at the group scale and PPV incidence at the scale of the individual tree. Most of the observed data fall below the curve corresponding to equation 1 . The estimate of effective sample size from equation 10 in Madden and Hughes (15) (using $\hat{\theta}=0.09$ ) was $\hat{v}=3.2$. The curve corresponding to equation 2 with $v=3.2$ provides a good description of the observed data (Fig. 2).

\section{DISCUSSION}

In the hierarchical sampling scheme, the sample covers $25 \%$ of the trees in a block. The sampling unit is a group of four trees in a two-by-two rectangular arrangement. In practice, three to four leaves are taken from each tree, one leaf from each main scaffold (16). Leaf material from each sampling unit is kept as a bulked sample. For subsequent laboratory assay, this bulked sample is divided into two subsamples of six to eight leaves each. If neither of the subsamples provides a PPV-positive ELISA result, the group is recorded as PPV-negative; otherwise the group is recorded as PPV-positive. Since only PPV incidence at the group scale is assessed in
Figure 2 shows the relationship between

this way, PPV incidence at the scale of the individual tree is then calculated from equation 3 , with $\hat{v}=3.2$.

The choice of a nominal group size of four trees reflects both the practicability of operating the scheme in the field and detecting PPV in the subsequent laboratory assay, as well as the statistical criteria required for a group testing procedure. When assessing the statistical criteria for group size and, in particular, the appropriateness of a group size of four as judged by these criteria, two issues predominate. The first is that estimates of incidence at the individual tree scale made from data collected at the group scale may be biased. The extent of the bias depends on the actual incidence of PPV (the true value of $\left.p_{\text {low }}\right)$, the number of groups, and the number of trees per group. The problem of bias tends to be more serious with a small number of groups and/or large numbers of trees per group (19). At the low levels of PPV incidence observed in Adams County, PA, the bias associated with estimates of PPV incidence made from the hierarchical sampling scheme is likely to be negligible. The second issue is that if group size is too large, all groups are likely to test positive even at small levels of $p_{\text {low }}$. It is desirable to choose a nominal group size that will result in some groups testing positive and some negative (4). At the low levels of PPV incidence observed in Adams County, a group size of four trees meets this requirement. These statistical considerations are not in conflict with the additional requirements relating to detection and practi-

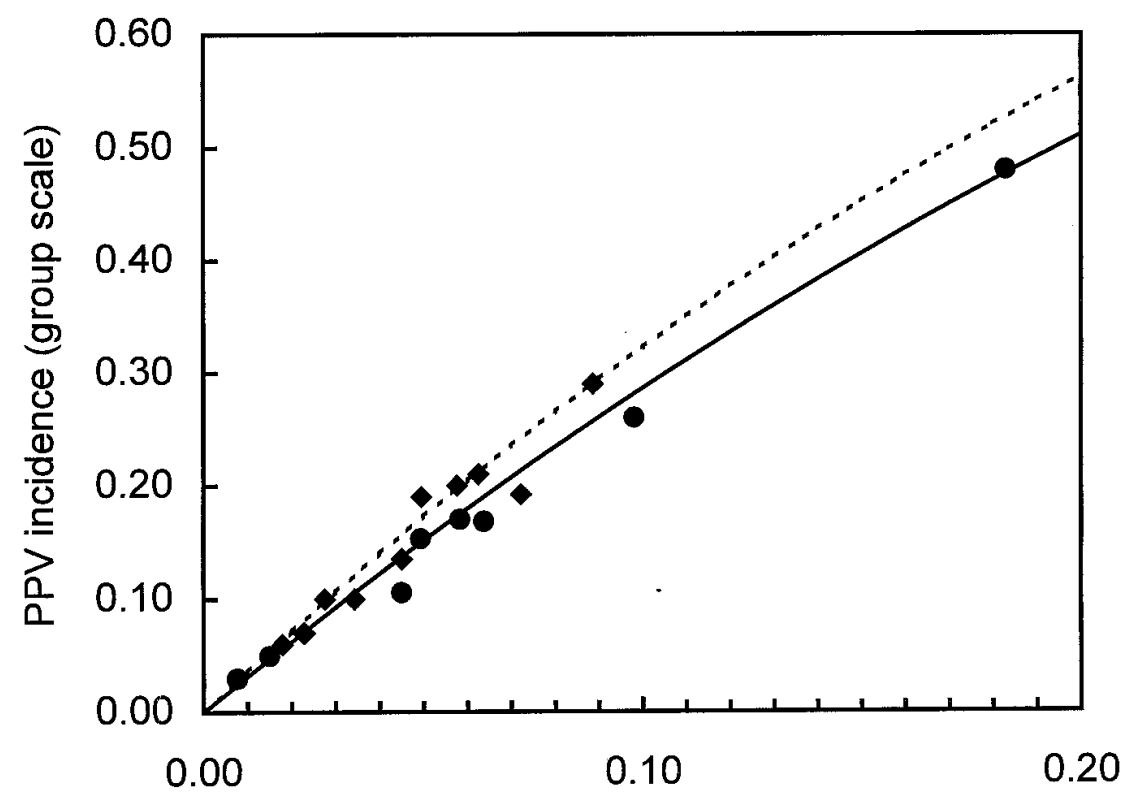

PPV incidence (tree scale)

Fig. 2. Relationship between incidence of Plum pox virus (PPV) at the group scale and its incidence at the individual tree scale. Each data point represents a PPV assessment in a stone fruit orchard block in Adams County, PA (•, assessed in fall 1999; $\bullet$, assessed in spring/summer 2000). The curve indicated by the dashed line (----) is based on equation 1 . The curve indicated by the solid line is based on equation 2 with $v=3.2$. cability mentioned above. If anything, the requirement for practicability of the method from the point of view of field operation will lead to the adoption of smaller group sizes than are strictly necessary from a statistical point of view.

PPV is known to be unevenly distributed within infected trees, and virus titer can fluctuate during a growing season. Nondetection problems may occur if the virus is not fully systemic at the time of sampling $(1,2)$ or because of low virus titer. If one or more PPV-infected trees in a group were not detected, the group may be falsely counted as PPV-negative. The chance of such false negatives occurring has to be considered when deciding on the policy implications of finding one or more PPVpositive groups in an orchard block. At the time of writing, the policy in Pennsylvania is that if a single PPV-positive group is found in a block, then the entire block is removed, including trees in groups that gave negative tests and those not tested. Even if detection were $100 \%$ accurate, there remains the possibility that an orchard block may be declared free of PPV infection when there is actually some (usually small) level of infection in the block. This occurs when, by chance, none of the PPV-infected trees present in the block are included in the sample. There is always a chance of such a misclassification when decision making is based on information obtained by sampling. This probability of reaching this type of false negative decision depends mainly on the true value of incidence and on sample size, and is shown by plotting the operating characteristic function of the sampling scheme (5-7). Orchard blocks that are declared free of PPV but are located in high-risk areas may be resampled at intervals to reduce the chance of missing an infection.

Of the 35 states that took part in the U.S. Plum Pox Virus National Surveillance Program during 2000 (most of which have adopted hierarchical sampling), Pennsylvania was the only one to report PPV-positive results. Of the rest, only PPV-negative samples were reported by 28 states and 6 had not made data available at the time of writing. It is therefore of interest to ask what conclusions can be drawn when only PPV-negative samples are reported. If the beta binomial is assumed to describe the distribution of infected plants per sampling unit, an approximate upper confidence interval for disease incidence can be determined when no infected plants are observed in samples $(16,17)$. The method described uses a negative binomial approximation of the beta-binomial distribution. Here we note an alternative method of obtaining an upper confidence interval for disease incidence, when no PPV-positive trees are observed, that employs the effective sample size $(\hat{v})$. An approximate one-sided $(1-P) \cdot 100 \%$ confidence interval from 0 to $p_{u}$, where $P$ is 
the designated significance level, is given by:

$$
p_{u} \approx 1-P^{\frac{1}{\hat{v} \cdot N}}
$$

This formula may be rearranged to show the number $(N)$ of groups of four trees, all PPV-negative, required to conclude with $(1-P) \cdot 100 \%$ confidence that the actual incidence of PPV is at most $p_{u}$ :

$$
N=\frac{\log (P)}{\hat{v} \cdot \log \left(1-p_{u}\right)}
$$

The values of $P$ and $p_{u}$ may be specified to meet regulatory requirements.

Important information on the species diversity and population dynamics of the vectors of PPV in Adams County, and in other areas that take part in the U.S. Plum Pox Virus National Surveillance Program, is still lacking. The value of effective sample size that we have calculated likely reflects the outcome of virus spread by a spectrum of aphid vector species, the range of which is not yet fully established. Further information on the vectors of PPV present in Adams County, and on their behavioral ecology, will help to establish the range over which the value $\hat{v}=3.2$ is applicable. If PPV is subsequently detected in areas where it is established that a different complement of aphid vector species is operating, a different value of effective sample size may need to be adopted for calculations of PPV incidence at the scale of the individual tree.

\section{ACKNOWLEDGMENTS}

The authors wish to express gratitude to the numerous individuals in the Pennsylvania Department of Agriculture, USDA APHIS, and Agdia Inc. who participated in the sample collection from PPV-infected blocks, serological processing of samples, and data entry procedures for the data set used in this study.

\section{LITERATURE CITED}

1. Adams, A. N. 1978. The incidence of plum pox virus in England and its control in orchards. Pages 213-219 in: Plant Disease Epidemiology. P. R. Scott and A. Bainbridge, eds. Blackwell Scientific Publications, Oxford.

2. Adams, A. N. 1978. The detection of plum pox virus in Prunus species by enzyme-linked immunosorbent assay. Ann. Appl. Biol. 90:215-221.

3. Cochran, W. G. 1977. Sampling Techniques. 3rd ed. John Wiley \& Sons, New York.

4. Hepworth, G. 1996. Exact confidence intervals for proportions estimated by group testing. Biometrics 52:1134-1146.

5. Hughes, G. 1999. Sampling for decision making in crop loss assessment and pest management: Introduction. Phytopathology 89:1080-1083.

6. Hughes, G., and Gottwald, T. R. 1998. Survey methods for assessment of citrus tristeza virus incidence. Phytopathology 88:715-723.

7. Hughes, G., and Gottwald, T. R. 1999. Survey methods for assessment of citrus tristeza virus incidence when Toxoptera citricida is the predominant vector. Phytopathology 89:487-494.

8. Hughes, G., and Madden, L. V. 1992. Aggregation and incidence of disease. Plant Pathol. 41:657-660.

9. Hughes, G., and Madden, L. V. 1993. Using the beta-binomial distribution to describe aggregated patterns of disease incidence. Phytopathology 83:759-763.

10. Hughes, G., and Madden, L. V. 1995. Some methods allowing for aggregated patterns of disease incidence in the analysis of data from designed experiments. Plant Pathol. 44:927943.

11. Hughes, G., McRoberts, N., Madden, L. V., and Gottwald, T. R. 1997. Relationships between disease incidence at two levels in a spatial hierarchy. Phytopathology 87:542-550.

12. Levy, L. 2000. ELISA laboratory protocols for the Plum Pox Virus National Surveillance Program. Published on-line. USDA, APHIS, PPQ.

13. Levy, L., Damsteegt, V., and Welliver, R. 2000. First report of Plum pox virus (Sharka disease) in Prunus persica in the United States. Plant Dis. 84:202.

14. Madden, L. V., and Hughes, G. 1995. Plant disease incidence: Distributions, heterogeneity, and temporal analysis. Annu. Rev. Phytopathol. 33:529-564.

15. Madden, L. V., and Hughes, G. 1999. An effective sample size for predicting plant disease incidence in a spatial hierarchy. Phytopathology 89:770-781.

16. Madden, L. V., and Hughes, G. 1999. Sampling for plant disease incidence. Phytopathology 89:1088-1103.

17. Madden, L. V., Hughes, G., and Munkvold, G. P. 1996. Plant disease incidence: Inverse sampling, sequential sampling, and confidence intervals when observed mean incidence is zero. Crop Prot. 15:621-632.

18. Poe, S. 2000. Background for the Plum Pox Virus National Surveillance Program. Published on-line. USDA, APHIS, PPQ.

19. Swallow, W. H. 1985. Group testing for estimating infection rates and probabilities of disease transmission. Phytopathology 75:882889.

20. Torrance, L., and Dolby, C. A. 1984. Sampling conditions for reliable routine detection by enzyme-linked immunosorbent assay for three ilarviruses in fruit trees. Ann. Appl. Biol. 104:267-276. 